Results The eHCI overall development score had the largest C-statistic when predicting all three cognitive development measures. For example, when predicting direct assessment numeracy at 6-9 years, eHCI overall development at 2-5 years had the strongest predictive ability (AUC 0.71, 95\% CI $0.69-0.73$ ), followed by eHCI numeracy (AUC 0.68, 95\% CI $0.65-0.70$ ) and direct assessment numeracy (AUC 0.67, 95\% CI $0.65-0.70)$.

Conclusion Child development at 2-5 years, as measured by the eHCI, was able to predict poor cognitive outcomes at 6-9 years in Lao PDR. Findings indicate we need not rely on costly, individual level direct assessment of early child development. Rather, an adult-reported tool such as the eHCI can be used to identify where supports and greater investments are required to promote children's later outcomes.

\section{OP14 FUNDING FOR PREVENTATIVE CHILDREN'S SERVICES AND RATES OF CHILDREN ENTERING CARE: A NATURAL EXPERIMENT USING LONGITUDINAL AREA-LEVEL DATA IN ENGLAND}

1Davara Bennett* ${ }^{2}$ Calum Webb, ${ }^{1}$ Kate Mason, ${ }^{1}$ Daniela Schlüter, ${ }^{1}$ Katie Fahy, ${ }^{1}$ Alexandros Alexiou, 'Sophie Wickham, 'Ben Barr, 'David Taylor-Robinson. 'Department of Public Health, Policy and Systems, University of Liverpool, Liverpool, UK; ${ }^{2}$ Department of Sociological Studies, University of Sheffield, Sheffield, UK

10.1136/jech-2021-SSMabstracts. 14

Background Children in state care face a range of adverse health outcomes, throughout the life course, relative to the general population. In England, over the last decade, the rate of children entering care has increased. The rate of change differs markedly for older and younger children, who may experience very different preventative services. Policies trailing the 2008 recession have led to inequitable reductions in spending on these services. Our aim was therefore to assess the impact of cuts to prevention on rates of preschool children and adolescents entering care between 2012 and 2019.

Methods Our outcomes were annual rates of children entering care across 150 English local authorities (LAs) (2012-19) for those aged 1-4, and young people aged 16-17. For the younger age group, count data were drawn from the Department for Education 'children looked after data return'. For the older age group, a Freedom of Information request yielded count data excluding unaccompanied children seeking asylum, whose care status will be largely unaffected by changes in our exposure of interest. Our exposure was agerelevant Children's Services prevention spend (2011-18), taken from LA expenditure outturns. Regression models were used to quantify, within LAs, associations between changing prevention spend and changing rates of children entering care the following year, while controlling for employment and child poverty rates. Models were estimated using the 'panelr' package, $\mathrm{R}$ version 3.6.3.

Results We found no association between changing prevention spend and changing rates of 1-4 year olds entering care. However, spending reductions were associated with rising rates for adolescents. We estimate that every $£ 10$ per child decrease in prevention spend was associated with an additional 1.9 per 100,000 children aged 16-17 entering care the following year (95\% CI 0.7, 2.9). According to our model, between 2012 and 2019, approximately 1 in 25 care entries in this age group were linked to the cuts.
Conclusion This study offers evidence that rising rates of older children entering care were partly driven by cuts to prevention services. These children face significant health and social risks in adulthood. Policies to tackle adverse trends in adolescent care entry should promote reinvestment in youth services, placing ordinary help on a robust statutory footing. We did not find comparable evidence for the younger age group, for whom rising poverty may be more important risk factors for care entry. Limitations, including issues relating to aggregate data, data quality and specification of causal lags, are assessed.

\section{OP15 HOUSING DISADVANTAGE IN CHILDHOOD AND HEALTH: A SYSTEMATIC REVIEW}

Yuxi Li*, Rebecca Bentley, Ankur Singh, Ludmila Fleitas Alfonzo. Melbourne School of Population and Global Health, The University of Melbourne, Melbourne, Australia

\subsection{6/jech-2021-SSMabstracts. 15}

Background Housing has been recognised as one of the most important determinants of health. While there is evidence that housing disadvantage can influence social and behavioural outcomes for children, little is known of the contribution of these pathways to children's health and wellbeing. This review aims to provide a synthesis of evidence from longitudinal cohort and interventional studies linking experiences of disadvantaged housing in childhood to health outcomes

Methods A literature search was performed on four databases including Medline (Ovid), EMBASE (Ovid), PsycINFO (Ovid), and Web of Science from 2000 to 2020. Peer-reviewed longitudinal studies assessing the association between housing disadvantage (physical quality, affordability, and instability) in childhood and subsequent physical and mental health were included. The methodological quality of selected studies was appraised using the ROBINS-I (Risk Of Bias In Non-randomised Studies - of Interventions) tool. A narrative synthesis was developed due to study heterogeneity.

Results Forty-five cohort studies met the inclusion criteria. The majority of the studies was evaluated to have a moderate risk of bias. The most studied housing exposure was residential mobility, followed by overcrowding and housing tenure. Other exposures examined in the set of eligible studies include housing facilities (e.g., ventilation, toilet), inadequate heating and self-rated housing condition. Most studies assessed multiple health outcomes, including mortality, respiratory health, substance misuse, subjective measures of health, diagnosed mental disorders, cardiovascular diseases risk factors, and healthcare utilisation. Across the studies, while many relationships remained mixed, consistent evidence of detrimental impact was identified between: poor housing conditions and mortality and self-rated health; inadequate heating and respiratory illness; poor ventilation and all-cause mortality; frequent residential moves and psychiatric mortality and morbidity. Little evidence is found between overcrowding in childhood and health outcomes.

Conclusion Evidence from longitudinal studies indicates that poor housing experience in childhood may impact health later in life. The findings confirm that housing as a key social determinant of child health, and interventions designed to mitigate housing disadvantage may have significant health gains across the life span. 